\title{
Panstrongylus megistus em ecótopos artificiais de ilhas do Alto Rio Paraná
}

\author{
Panstrongylus megistus in artificial ecotopes on islands of the Paraná River
}

\author{
Ana Lucia Falavigna Guilherme ${ }^{1}$, Elias Seixas Lorosa ${ }^{2}$, Arnaldo Lima Costa ${ }^{3}$, \\ Gilberto Cezar Pavanelli ${ }^{1}$ e Silvana Marques de Araújo ${ }^{4}$
}

\begin{abstract}
Resumo Em resposta a denúncias de triatomíneos em ilhas do Alto Rio Paraná foram investigados 145 ecótopos artificiais e $4(2,8 \%)$ deles encontravam-se infestados: residência, "clube", ex-escola e monte de madeira. Foram analisados 17 de 35 P. megistus coletados; 12 (70,6\%) apresentavam-se infectados por Trypanosoma cruzi. Ave e roedor constituíram as fontes alimentares mais comuns. Todos os exames sorológicos (56 de humanos, 18 de cães e 10 de gatos) foram negativos.
\end{abstract}

Palavras-chaves: Ilhas. Ecótopo artificial. Panstrongylus megistus. Trypanosoma cruzi. Soroprevalência.

\begin{abstract}
In response to complaints of triatomines on islands of the Alto Paraná River, an investigation to determine the presence of triatomines was conducted in 145 artificial ecotopes and 4 (2.8\%) were infested, comprising a residence, a sports and leisure club, a former school and a woodpile. Of the $35 \mathrm{P}$. megistus collected, 17 were analyzed and 12 (70.6\%) were found to be infected with Trypanosoma cruzi. Birds and rodents were the most common food sources. All serologic examinations (56 human, 18 dogs and 10 cats) were negative.
\end{abstract}

Key-words: Islands. Artificial ecotopes. Panstrongylus megistus. Trypanosoma cruzi. Seroprevalence.

As ilhas pertencem à Marinha e são amparadas por leis de proteção ambiental. Mesmo assim, grande parte delas encontra-se devastada, a exemplo da Ilha de Santa Catarina, em Santa Catarina, onde encontrou-se Panstrongylus megistus domiciliado com altos índices de positividade para Trypanosoma cruzi ${ }^{10}$. Na llha Mosqueiro, Pará, verificou-se um caso autóctone de doença de Chagas ${ }^{9}$.

De acordo com a FUNASA/Porto Rico/PR, nunca foi realizado um controle sistemático de triatomíneos nas ilhas do Alto Rio Paraná, embora tenham sido registradas denúncias da ocorrência desses insetos em quatro delas. Várias famílias se apossaram dessas ilhas ao longo dos anos e utilizaram-nas como fonte de subsistência pelo cultivo de milho, de ginseng, da pecuária bovina e suína e da pesca artesanal. Na ilha Mutum, há 50 posses e 30 famílias; na ilha Óleo Cru, 70 posses e 45 famílias; na ilha Japonesa, 9 posses e 3 famílias e na ilha Cajá, apenas 4 posses e 1 família. Não há energia elétrica e no pulso das cheias algumas casas são inundadas. Essas ilhas estão localizadas entre os Estados de São Paulo, Mato Grosso do Sul e Paraná (Figura 1). Sua maior proximidade é com o lado continental do Estado do Paraná, inclusive no acesso aos serviços disponíveis nas cidades.

Segundo Wanderley ${ }^{11}$, o controle da transmissão da doença de Chagas no Estado de São Paulo não constitui problema de saúde pública, com a eliminação do Triatoma infestans, sendo que há espécies da região silvestre local, como o $P$. megistus, para o qual tem se verificado aproximação cada vez maior às moradias ${ }^{4}$.

Segundo a FUNASA/PR, o último relato do encontro da espécie $T$. infestans na região noroeste do Paraná foi em 1986. Falavigna et $\mathrm{al}^{3}$ observaram a presença de Triatoma sordida e P. megistus no peridomicílio e até mesmo em alguns domicílios de municípios do noroeste do Estado do Paraná.

1. Curso de Pós-graduação em Ecologia de Ambientes Aquáticos Continentais da Universidade Estadual de Maringá (UEM), Maringá, PR. Brasil. 2. Laboratório de Entomologia 2. Fundação Oswaldo Cruz, Rio de Janeiro, RJ. 3. Fundação Nacional de Saúde/Subdistrito de Maringá, PR. 4. Departamento de Análises Clínicas da Universidade Estadual de Maringá, Maringá, PR.

Apoio financeiro: Fundação Nacional de Saúde, convênio FUNASA/UEM № 1899/98 e Curso de Pós-graduação em Ecologia de Ambientes Aquáticos Continentais - Universidade Estadual de Maringá.

Endereço para correspondência: Drª Ana Lucia Falavigna Guilherme. Curso de Pós-graduação/PEA/UEM. Av. Colombo 5790, $87020-900$ Maringá, PR, Brasil. Recebido para publicação em 24/11/2000. 
A presente investigação preocupou-se com a ocorrência de triatomíneos em ecótopos artificiais, sua taxa de infecção por T. cruzi e sua fonte alimentar, a prevalência de infecção por T. cruzi em humanos e em animais domésticos nas ilhas Mutum, Óleo Cru, Japonesa e Cajá (Figura 1).

Durante o mês de fevereiro de 2000, inspecionouse os ecótopos artificiais de no mínimo $18 \%$ do total de posses de cada ilha, ou seja, unidades domiciliares (Uds) constituídas de domicílio e peridomicílio, quer estivessem ou não habitadas ou que fossem habitadas temporariamente (clubes). Foram priorizadas as Uds onde ocorreu denúncia de triatomíneos junto a FUNASA/Porto Rico/PR, as circunvizinhas e as Uds que fossem habitadas.

Foram considerados positivos os ecótopos onde ninfas, adultos e/ou ambos haviam sido encontrados. Os ecótopos positivos foram borrifados posteriormente com cipermetrina (125mg i.a. $/ \mathrm{m}^{2}$ ). Parte dos triatomíneos coletados foi enviada ao laboratório de Parasitologia Básica da UEM com a finalidade de se verificar a sua infecção por T. cruzi. Foi também realizado o teste de precipitina ${ }^{8}$ pelo Laboratório de Entomologia da FIOCRUZ/RJ utilizando os anti-soros anti-ave, cão, gato, cabra ou boi, homem, gambá, cavalo, porco, roedor, sapo e lagarto?

Uma amostra de sangue foi colhida dos ilhéus, de cães e gatos em papel de filtro. O material foi submetido à reação de imunoflorescência indireta (IFI) segundo Camargo et $\mathrm{al}^{1}$, utilizando-se antígenos de T. cruzi marca Biolab e conjugados específicos para homem (Biolab), cão e gato (Sigma).

$\mathrm{Na}$ llha Óleo Cru, os triatomíneos foram encontrados em dois ecótopos (Tabela 1). Um no interior do domicílio, sendo que o morador havia encontrado outro exemplar sugando-o dias antes. Outro em um "clube", dentro de um armário no quarto. Na Ilha Japonesa foram encontrados triatomíneos em uma ex-escola que tem servido de abrigo para bombeiros, pescadores, visitantes, entre outros. Na Ilha Mutum foi encontrado um triatomíneo em um monte de madeira próxima à casa. Todas os ecótopos positivos já haviam sido positivos para $P$. megistus em outras épocas, segundo FUNASA/Porto Rico/ PR.

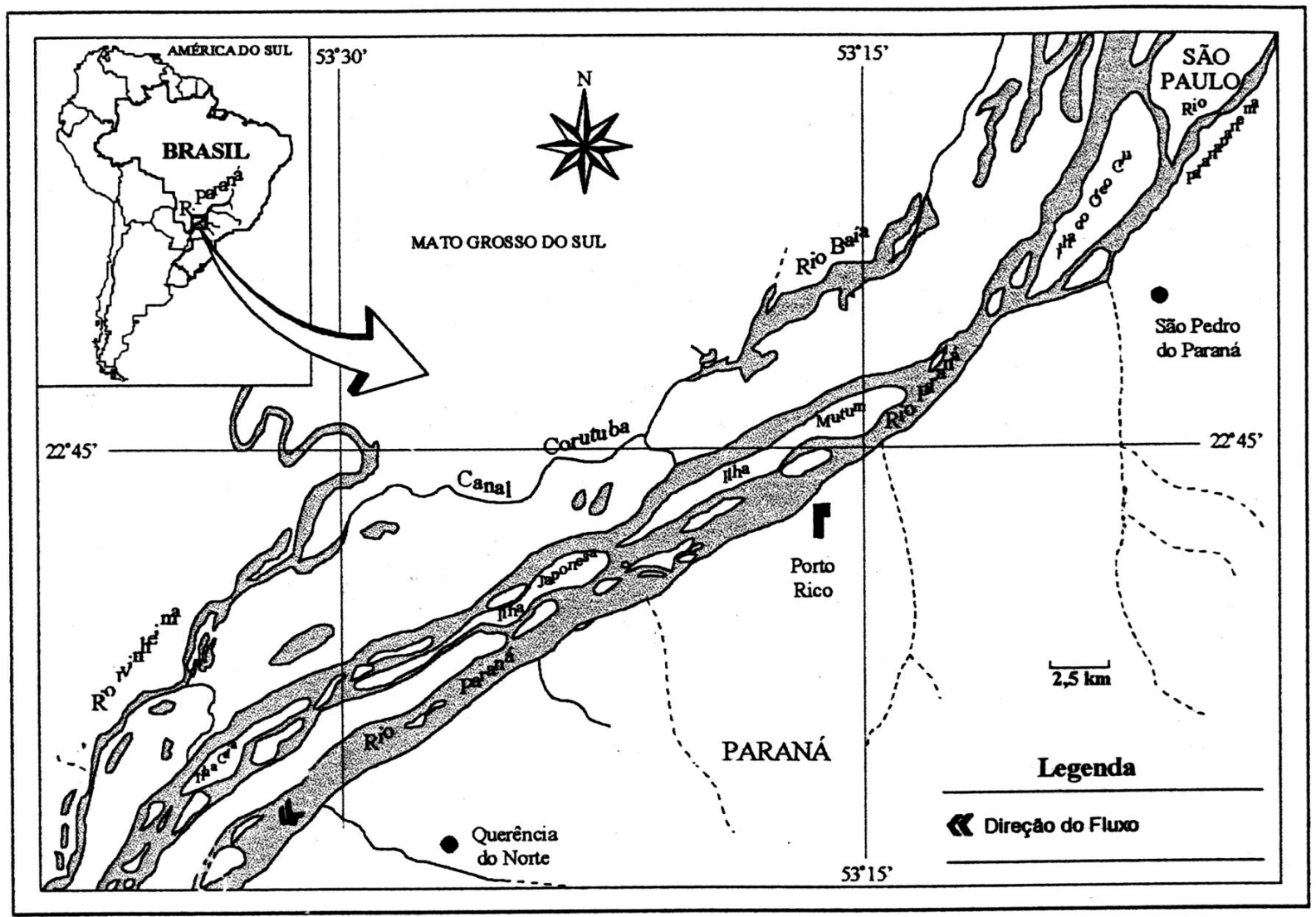
Figura 1- Trecho do Alto Rio Paraná compreendido entre os municípios de Querência do Norte e São Pedro do Paraná,
Paraná, Brasil onde estão localizadas as ilhas em que foi realizada investigação triatomínica, em fevereiro de 2000. 
Tabela 1- Investigação triatomínica realizada em ecótopos artificiais de unidades domiciliares (Uds) existentes em ilhas do Alto Rio Paraná e sua positividade para T. cruzi, durante o mês de fevereiro de 2000.

\begin{tabular}{|c|c|c|c|c|c|c|c|c|c|c|}
\hline \multirow{4}{*}{ Ilha } & & & \multicolumn{3}{|c|}{ Ecótopos artificiais } & \multicolumn{5}{|c|}{ P. megistus } \\
\hline & \multirow{2}{*}{\multicolumn{2}{|c|}{$\begin{array}{l}\text { Uds existentes/ } \\
\text { Uds investigadas }\end{array}$}} & \multirow{2}{*}{\multicolumn{2}{|c|}{$\begin{array}{l}\text { investigados/ } \\
\text { infestados }\end{array}$}} & \multirow{3}{*}{ ecótopo positivo } & \multirow{3}{*}{ ninfa } & \multirow{3}{*}{ adulto } & \multirow{3}{*}{ total } & \multirow{2}{*}{\multicolumn{2}{|c|}{$\begin{array}{l}\text { analisados/ } \\
\text { infectados }\end{array}$}} \\
\hline & & & & & & & & & & \\
\hline & $\mathrm{n}^{\circ}$ & $\%$ & $\mathrm{n}^{0}$ & $\%$ & & & & & № & $\%$ \\
\hline Mutum & $50 / 11$ & 22,0 & $57 / 01$ & 1,8 & monte madeira & 0 & 1 & 1 & $01 / 00$ & 00,0 \\
\hline \multirow[t]{2}{*}{ Óleo Cru } & $70 / 13$ & 18,6 & $58 / 02$ & 3,4 & domicílio & 3 & 2 & 5 & 02/01 & 50,0 \\
\hline & & & & & "clube" & 2 & 2 & 4 & $00 / 0$ & - \\
\hline Japonesa & 09/06 & 66,7 & $17 / 01$ & 5,9 & ex-escola & 9 & 6 & 25 & $114 / 11$ & 78,6 \\
\hline Cajá & $04 / 04$ & 100,0 & $13 / 00$ & 0,0 & - & 0 & 0 & 0 & - & - \\
\hline Total & $133 / 34$ & 25,6 & $145 / 04$ & 2,8 & - & 14 & 11 & 35 & $117 / 12$ & 70,6 \\
\hline
\end{tabular}

De 17 P. megistus analisados, 12 (70,6\%) estavam positivos para T. cruzi (Tabela 1), sendo encontrados em ambientes freqüentados pelo homem. Do conteúdo intestinal dos exemplares positivos, cinco foram reativos para sangue de ave, cinco para sangue de roedor; um para sangue de ave/roedor e um para sangue de ave/cão. Foram realizadas 56 reações sorológicas para humanos sendo $21(37,5 \%)$ de menores de 15 anos. Dezoito reações sorológicas para cães e 10 para gatos. Nenhuma foi reativa para T. cruzi.

Pelos resultados pode-se afirmar a existência de ciclo silvestre e ausência do ciclo domiciliar nestas ilhas. Segundo Silveira \& Rezende ${ }^{6}$, na região sul o $P$. megistus está presente em ecótopos silvestres, invade apenas esporadicamente a casa e constitui colônias no domicílio muito raramente. A discussão que emerge deste ponto é que apesar da devastação ocorrida nestas ilhas, o homem com suas Uds precárias vive inserido no contexto silvestre como se ele fosse parte deste ambiente. Embora várias destas ilhas estejam devastadas, algumas estão reassumindo características bastante silvestres, a exemplo das ilhas Cajá e Japonesa. Isto deve-se a intensa fiscalização do IBAMA proibindo não só a pecuária extensiva como novas construções na região.

Chama a atenção a desmotivação e o "não sentirse dono" que os indivíduos têm com relação às suas habitações, incluindo os "clubes", casas simples partilhadas por grupos de amigos, muitas vezes com péssima infra-estrutura. Isto tem proporcionado alta rotatividade de pessoas na área, sem o devido cuidado com a moradia. Além disso, há outros fatores como precárias condições de higiene, pobreza, presença de grande número de animais domésticos e o expressivo desconhecimento sobre o triatomíneo, tanto do ilhéu quanto do turista que, segundo Silveira \& Vinhaes ${ }^{7}$, podem levar a considerar estas populações sob risco de contaminação.

Apesar de reduzido número de ecótopos artificiais com a presença de P. megistus (4/145), 70,6\% dos triatomíneos analisados estavam positivos para T. cruzi. Diferentes índices de infecção têm sido observados em $P$. megistus coletados em ecótopos artificiais, como por exemplo, $56,5 \%$ na ilha de Santa Catarina $^{10}$ e $2,7 \%$ para $P$. megistus coletados em vários locais do país ${ }^{7}$. A elevada taxa de positividade encontrada no presente trabalho pode ser atribuída, em parte, ao reduzido tamanho da amostra. Contudo, para espécies de triatomíneos que são originárias da própria área silvestre e tenham alta capacidade vetorial, como o P. megistus e o Triatoma brasiliensis, é necessário conter a pressão de reinfestação mesmo quando os triatomíneos estejam limitados a nível peridomiciliar? ${ }^{7}$.

Em todos os ecótopos artificiais positivos, com exceção da ilha Mutum, foram encontrados ninfas e adultos. Estes achados concordam com os de Forattini et $\left.a\right|^{5}$ que verificaram na região sudeste do Brasil maior dispersão das formas adultas de $s P$. megistus entre os meses de outubro e dezembro, sendo que a partir destes meses a população já é constituída de ninfas e adultas.

É importante considerar o fato de não estar ocorrendo transmissão ativa na área em estudo, e a fonte de infeção para os triatomíneos positivos pode estar relacionada principalmente a roedores. Nenhum triatomíneo analisado alimentou-se de sangue humano. Todavia, não pode ser negligenciado o caso de um paciente chagásico de 35 anos, freqüentador assíduo há dez anos da llha Mutum, em atividades de lazer. Segundo informações do próprio indivíduo a única possibilidade de ter se contaminado seria as pernoites em "clubes" abandonados desta ilha, já que sempre residiu na cidade de Maringá, em moradia de boa qualidade, não possuindo antecedentes familiares ou transfusionais que se relacionassem à infecção.

Os ilhéus carecem de melhor infra-estrutura, profissionalização e esclarecimentos, sendo a população formada por pessoas que sobrevivem com salários miseráveis, isto quando têm a oportunidade de serem remunerados pelo seus serviços, considerando ainda que no pulso das cheias algumas famílias têm que abandonar suas casas. Nestas condições, os achados aqui relatados não devem ser desconsiderados, principalmente neste momento em que o Brasil prepara-se para declarar como controlada a transmissão vetorial da doença de Chagas. 


\section{REFERÊNCIAS BIBLIOGRÁFICAS}

1. Camargo M, Silva GR, Castilho EA, Silveira AC. Inquérito sorológico da infecção chagásica no Brasil, 1975/1980. Revista do Instituto de Medicina Tropical de São Paulo 26: 192-204, 1984.

2. Diotaiuti L, Azeredo BV, Busek SCU, Fernandes AJ. Controle do Triatoma sordida no peridomicílio rural do município de Porteirinha, Minas Gerais, Brasil. Revista Panamericana de Salud Pública 3: 21-25, 1998.

3. Falavigna-Guilherme AL, Pavanelli GC, Costa AL, Araújo SM. Prevalence and infections in triatomines captured in artificial ecotopes in municipalities under epidemiological surveillance in northwest Paraná, Brazil. Revista Panamericana de Salud Pública 9:385-392, 2001.

4. Ferraz Filho NA, Rodrigues VLCC. Distribuição e índice de infecção natural de triatomíneos capturados na região de Campinas, São Paulo, Brasil. Revista da Sociedade Brasileira de Medicina Tropical 20: 25-30, 1987.

5. Forattini OP, Santos JLF, Ferreira AO, Silva EOR, Rabello EX. Aspectos ecológicos da tripanossomíase americana. XVI- Dispersão e ciclos anuais de colônias de Triatoma sordida e de Panstrongylus megistus espontaneamente desenvolvidas em ecótopos artificiais. Revista de Saúde Pública de São Paulo 13: 299-313, 1979.
6. Silveira AC, Rezende DF. Epidemiologia e controle da transmissão vetorial da doença de Chagas no Brasil. Revista da Sociedade Brasileira de Medicina Tropical 27 (supl III): 11-22, 1994.

7. Silveira AC, Vinhaes M. Doença de Chagas: aspectos epidemiológicos e de controle. Revista da Sociedade Brasileira de Medicina Tropical 31 (supl II): 15-60, 1998.

8. Siqueira, A F. Estudos sobre a reação de precipitina aplicada a identificação de sangue ingerido por triatomíneos. Revista do Instituto de Medicina Tropical de São Paulo 2: 41-53, 1960.

9. Souza AAA, Silveira FT, Miles MA, Póvoa MM, Lima JAN, Valente SAS. Epidemiologia de um caso de doença de Chagas na llha do Mosqueiro-Pará. Revista da Sociedade Brasileira de Medicina Tropical 21: 187-192, 1988.

10. Steindel M, Toma HK, Carvalho-Pinto CJ, Grisard EC, Schlemper BR. Colonization of artificial ecotopes by Panstrongylus megistus in Santa Catarina island, Florianópolis, Santa Catarina, Brazil. Revista do Instituto de Medicina Tropical de São Paulo 36: 43-50, 1994.

11. Wanderley DMV. Perspectivas de controle da doença de Chagas no Estado de São Paulo. Tese de Doutorado, Faculdade de Saúde Pública da Universidade de São Paulo, São Paulo, 1994. 